International Journal of Pure and Applied Mathematics

Volume 82 No. 4 2013, 547-555

ISSN: 1311-8080 (printed version); ISSN: 1314-3395 (on-line version)

url: http://www.ijpam.eu

doi: http://dx.doi.org/10.12732/ijpam.v82i4.4

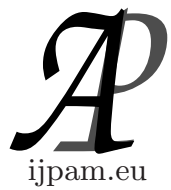

\title{
ANALYTIC SOLUTION OF \\ A NONLINEAR BLACK-SCHOLES EQUATION
}

\author{
Joseph Eyang'an Esekon \\ Department of Statistics and Actuarial Science \\ Maseno University \\ P.O. Box 333, Maseno, KENYA
}

\begin{abstract}
We study a nonlinear Black-Scholes partial differential equation whose nonlinearity is as a result of transaction costs that lead to market illiquidity. After reducing the equation into a nonlinear parabolic porous medium type equation, we find that the assumption of a traveling wave profile to the porous medium type equation reduces it further to ordinary differential equations. Solutions to all these transformed equations together with the use of localizing boundary conditions facilitate a twice continuously differentiable solution to the nonlinear Black-Scholes equation. We also find that the option is always more volatile compared to the stock. All the risk parameters except Gamma are negative throughout time $t$.
\end{abstract}

AMS Subject Classification: $35 \mathrm{~K} 10,35 \mathrm{~K} 55$

Key Words: porous medium equation, analytic solution, illiquid markets, transaction cost

\section{Introduction}

Two primary assumptions are used in formulating classical arbitrage pricing theory: frictionless and competitive markets. There are no transaction costs and restrictions on trade in a frictionless market. In a competitive market, a trader can buy or sell any quantity of a security without changing its price.

Received: July 16, 2012

(c) 2013 Academic Publications, Ltd. url: www.acadpubl.eu 
The notion of liquidity risk is introduced on relaxing the assumptions above.

The purpose of this paper is to obtain an analytic solution of the nonlinear Black-Scholes equation arising from transaction costs by Cetin et al. in [3]. This is done by differentiating the equation twice with respect to the stock price. After substitutions and transformations, we get a nonlinear parabolic porous medium type equation. Assuming a traveling wave solution to the porous medium type equation reduces it further to ordinary differential equations (ODEs). All these transformed equations are solved to obtain an analytic solution to the nonlinear Black-Scholes equation. A thorough analysis of the Black-Scholes equation is found in [4].

This paper is organized as follows. Section 2 describes the modified option pricing theory. The solution to the nonlinear Black-Scholes equation is presented in Section 3. The Greek parameters are studied in Section 4. Section 5 concludes the paper.

\section{Modified Option Valuation Model}

\subsection{Cetin et al. (2004)}

In this work, we will consider the continuous-time transaction-cost model for illiquid markets by Cetin et al. in [3]. Two assets are used in the model: a bond (or a risk-free money market account with spot rate of interest $r \geq 0$ ) whose value at time $t$ is $B_{t} \equiv 1$, and a stock. The stock is assumed to be risky and illiquid while the bond is assumed to be riskless and liquid.

In the transaction-cost model, a fundamental stock price process $S_{t}^{0}$ follows the dynamics

$$
d S_{t}^{0}=\mu S_{t}^{0} d t+\sigma S_{t}^{0} d W_{t}, \quad 0 \leq t \leq T
$$

where $\mu$ is drift, $\sigma$ is volatility, $T$ is time to expiry, and $W_{t}$ is the Wiener process. When trading $\alpha$ shares, the transaction price to be paid at time $t$ is

$$
\bar{S}_{t}(\alpha)=e^{\rho \alpha} S_{t}^{0}, \quad \alpha \in \mathbb{R},
$$

where $\rho$ is a liquidity parameter with $\rho \geq 0$. It is obvious from equation (2.1) that if $\rho>0$ the trader will pay more than the fundamental price when buying the stock (i.e. $\alpha>0$ ) and get less when selling (i.e. $\alpha<0$ ). Therefore, a bid-ask-spread with size depending on $\alpha$ is essentially modeled by (2.1).

Taking the function $\phi\left(t, S_{t}^{0}\right)$ to be the number of shares traded rather than held and identifying this function to be a trading strategy, then for a Markovian trading strategy $\Phi_{t}=\phi\left(t, S_{t}^{0}\right)$ for a smooth function $\phi=u_{S}$, we have $\phi_{S}=u_{S S}$. 
If the stock and bond positions are $\Phi_{t}$ and $\beta_{t}$ respectively where $\Phi_{t}$ is a semimartingale, then the paper value is given by

$$
V_{t}^{M}=\Phi_{t} S_{t}^{0}+\beta_{t} .
$$

The change in the quadratic variation,

$$
\langle\Phi\rangle t=\int_{0}^{t}\left(\phi_{S}\left(\tau, S_{\tau}^{0}\right) \sigma S_{\tau}^{0}\right)^{2} d \tau,
$$

is given by $d\langle\Phi\rangle t=\left(u_{S S}\left(t, S_{t}^{0}\right) \sigma S_{t}^{0}\right)^{2} d t$ since $\phi_{S}=u_{S S}$.

Applying Itô's formula to $u\left(t, S_{t}^{0}\right)$ gives

$$
d u\left(t, S_{t}^{0}\right)=u_{S}\left(t, S_{t}^{0}\right) d S_{t}^{0}+\left(u_{t}\left(t, S_{t}^{0}\right)+\frac{1}{2} \sigma^{2}\left(S_{t}^{0}\right)^{2} u_{S S}\left(t, S_{t}^{0}\right)\right) d t .
$$

Theorem A3 of [3] yields the wealth dynamics of a self-financing strategy as

$$
\begin{aligned}
d V_{t}^{M} & =\Phi_{t} d S_{t}^{0}-\rho S_{t}^{0} d\langle\Phi\rangle t \\
& =u_{S}\left(t, S_{t}^{0}\right) d S_{t}^{0}-\rho \sigma^{2}\left(u_{S S}\left(t, S_{t}^{0}\right)\right)^{2}\left(S_{t}^{0}\right)^{3} d t
\end{aligned}
$$

since $\Phi_{t}=\phi\left(t, S_{t}^{0}\right)=u_{S}$ and $d\langle\Phi\rangle t=\left(u_{S S}\left(t, S_{t}^{0}\right) \sigma S_{t}^{0}\right)^{2} d t$. Uniqueness of semimartingale decompositions means that the $d t$-terms in (2.2) and (2.3) must coincide. Equate these $d t$-terms. Applying a little algebra and then simplifying we get

$$
u_{t}+\frac{1}{2} \sigma^{2} S^{2} u_{S S}\left(1+2 \rho S u_{S S}\right)=0, \quad u(S, T)=h\left(S_{T}\right),
$$

where $h\left(S_{T}\right)$ is a terminal claim whose hedge cost, $u\left(S_{t}, t\right)$, is the solution to (2.4). For instance the terminal condition for a European call option is given by

$$
u(S, T)=\max \{S-K, 0\} \quad \text { for } \quad S \geq 0,
$$

where $K>0$ is the striking price. The boundary conditions for the option are as follows:

$$
\begin{gathered}
u(0, t)=0 \quad \text { for } \quad 0 \leq t \leq T, \\
u(S, t) \sim S-K e^{-r(T-t)} \quad \text { as } \quad S \rightarrow \infty .
\end{gathered}
$$

We take the last condition to mean that

$$
\lim _{S \rightarrow \infty} \frac{u(S, t)}{S-K e^{-r(T-t)}}=1
$$

uniformly for $0 \leq t \leq T$.

The magnitude of the market impact is determined by $\rho S$. Large $\rho$ implies a big market impact of hedging. If $\rho=0$, the asset's price follows the standard Black-Scholes model in [2] with constant volatility $\sigma$. 


\subsection{Bakstein and Howison (2003)}

A model for an illiquid market which results in the following PDE,

$$
u_{t}+\frac{1}{2} \sigma^{2} S^{2} u_{S S}\left(1+2 \rho S u_{S S}\right)+\frac{1}{2} \rho^{2}(1-\alpha)^{2} \sigma^{2} S^{4} u_{S S}^{3}+r S u_{S}-r u=0
$$

where $\rho \in \mathbb{R}$ is a measure of the liquidity of the market and $\alpha$ is a measure of the price slippage impact of a trade felt by all market participants was developed in Theorem 3.1 of [1].

When $\alpha=1$, this corresponds to no slippage and equation (2.6) reduces to the model whose PDE is given by

$$
u_{t}+\frac{1}{2} \sigma^{2} S^{2} u_{S S}\left(1+2 \rho S u_{S S}\right)+r S u_{S}-r u=0, \quad u(S, T)=h\left(S_{T}\right) .
$$

This is the Cetin et al. (2004) model given in (2.4) with $r>0$.

\section{Solution to the Nonlinear Black-Scholes Equation}

Lemma 3.0.1. If $\nu(\xi)$ is a twice continuously differentiable function, and $x$ and $t$ are the spatial and time variables respectively, then there exists a traveling wave solution to the porous medium type equation,

$$
V_{t}+\frac{\sigma^{2}}{2}\left(V V_{x}+\frac{1}{2} V^{2}+\frac{2 r}{\sigma^{2}} V\right)_{x}=0 \quad \text { in } \quad \mathbb{R} \times[0, \infty),
$$

of the form

$$
V(x, t)=\nu(\xi) \quad \text { where } \xi=x-c t, \quad \xi \in \mathbb{R}
$$

for $\sigma>0$ and $r \geq 0$ such that $V=V(x, t)$ is a traveling wave of permanent form which translates to the right with constant speed $c>0$.

Proof. Applying the chain rule to (3.2) gives

$$
V_{t}=-c \nu^{\prime}(\xi), \quad V_{x}=\nu^{\prime}(\xi), \quad \text { and } \quad V_{x x}=\nu^{\prime \prime}(\xi),
$$

where the prime denotes $\frac{d}{d \xi}$. Substituting these expressions into (3.1), we conclude that $\nu(\xi)$ must satisfy the nonlinear second order ODE

$$
-c \nu^{\prime}+\frac{\sigma^{2}}{2}\left(\nu \nu^{\prime \prime}+\left(\nu^{\prime}\right)^{2}+\nu \nu^{\prime}+\frac{2 r}{\sigma^{2}} \nu^{\prime}\right)=0 \quad \text { in } \quad \mathbb{R}
$$

and hence $V$ solves (3.1).

Assume also that the travelling wave solution $V(x, t)$ is localized. Then

$$
\lim _{x \rightarrow \pm \infty} V(x, t)=\lim _{x \rightarrow \pm \infty} V_{x}(x, t)=\lim _{x \rightarrow \pm \infty} V_{x x}(x, t)=0
$$


or from (3.2) the localizing boundary conditions (3.4) become

$$
\lim _{\xi \rightarrow \pm \infty} \nu(\xi)=\lim _{\xi \rightarrow \pm \infty} \nu^{\prime}(\xi)=\lim _{\xi \rightarrow \pm \infty} \nu^{\prime \prime}(\xi)=0 .
$$

We can now solve (3.3) in a closed-form by first writing it as

$$
\frac{d}{d \xi}\left(\frac{\sigma^{2}}{2} \nu \nu^{\prime}+\frac{\sigma^{2}}{4} \nu^{2}-c \nu+r \nu\right)=0 .
$$

Integrating (3.6) and rearranging gives the standard form (see [6])

$$
\nu^{\prime}=\frac{2}{\sigma^{2} \nu}\left(c \nu-r \nu-\frac{\sigma^{2}}{4} \nu^{2}+\kappa\right)
$$

where $\kappa$ is a constant of integration. From the localizing boundary conditions (3.5), $\kappa=0$. Simplifying (3.7) further we realize that $\nu(\xi)$ satisfies the first order linear autonomous and separable ODE

$$
-2 \frac{d \nu}{d \xi}=\nu-\frac{4}{\sigma^{2}}(c-r) .
$$

Rearranging the equation above and integrating gives

$$
\nu(\xi)=e^{\frac{\xi_{0}-\xi}{2}}+\frac{4}{\sigma^{2}}(c-r) \quad \text { for } \quad c, \sigma>0, r \geq 0, \quad \text { and } \quad x \in \mathbb{R},
$$

where $\xi_{0}$ is a constant of integration. Hence,

$$
V(x, t)=e^{\frac{x_{0}-(x-c t)}{2}}+\frac{4}{\sigma^{2}}(c-r)
$$

for $c, \sigma>0, r, t \geq 0$, and $x \in \mathbb{R}$, where $x_{0}$ is a constant of integration given by

$$
\xi_{0}=x_{0}-c \cdot 0=x_{0}
$$

as $t=0$.

Theorem 3.0.2. If $V(x, t)$ is any positive solution to the porous medium type equation

$$
V_{t}+\frac{\sigma^{2}}{2}\left(V V_{x}+\frac{1}{2} V^{2}+\frac{2 r}{\sigma^{2}} V\right)_{x}=0 \quad \text { in } \quad \mathbb{R} \times[0, \infty),
$$

then

$$
u(S, t)=S-\frac{\sqrt{S_{0}}}{\rho}\left(\sqrt{S} e^{\left(\frac{r+\sigma^{2} / 4}{2}\right) t}+\frac{\sqrt{S_{0}}}{4} e^{\left(r+\sigma^{2} / 4\right) t}\right)
$$

solves the nonlinear Black-Scholes equation

$$
u_{t}+\frac{1}{2} \sigma^{2} S^{2} u_{S S}\left(1+2 \rho S u_{S S}\right)+r S u_{S}-r u=0, \quad u(S, T)=h\left(S_{T}\right)
$$

for $S_{0}, S, \sigma, \rho>0$ and $r, t \geq 0$, where $S_{0}$ is the initial stock price. 
Proof. To obtain the solution to equation (3.10) we differentiate it twice with respect to $S$ to get

$$
\begin{aligned}
& w_{t}+\frac{\sigma^{2} S^{2}}{2}(1+4 \rho S w) w_{S S}+2 \rho \sigma^{2} S^{3} w_{S}^{2}+2 \sigma^{2} S(1+6 \rho S w) w_{S}+r S w_{S} \\
& +\sigma^{2}(1+6 \rho S w) w+r w=0 \quad \text { in } \quad \mathbb{R}_{+} \times[0, \infty),
\end{aligned}
$$

where $w=u_{S S}$.

Applying the transformations $w=\frac{v}{\rho S}$ and $x=\ln S$ to the reaction-advectiondiffusion equation above and simplifying we get

$$
v_{t}+\frac{\sigma^{2}}{2}(1+4 v) v_{x x}+2 \sigma^{2} v_{x}^{2}+\frac{\sigma^{2}}{2}(1+4 v) v_{x}+r v_{x}=0 \quad \text { in } \quad \mathbb{R} \times[0, \infty) .
$$

If we let $v=\frac{V-1}{4}$ then (3.11) reduces to equation (3.1).

Hence, substituting $v=\frac{V-1}{4}$ into (3.8) gives the solution to (3.11) as

$$
v(x, t)=\frac{1}{4} e^{\frac{x_{0}-(x-c t)}{2}}+\frac{1}{\sigma^{2}}(c-r)-\frac{1}{4}
$$

for $c, \sigma>0, r, t \geq 0$, and $x, x_{0} \in \mathbb{R}$. Substituting $w=\frac{v}{\rho S}$ and $x=\ln S$ into (3.12) gives

$$
u_{S S}=\frac{1}{\rho S}\left(\frac{1}{4} \sqrt{\frac{S_{0}}{S}} e^{\frac{c t}{2}}+\frac{1}{\sigma^{2}}(c-r)-\frac{1}{4}\right)
$$

for $\rho, c, S, \sigma>0$, and $r, t \geq 0$. Since $\ln S_{0}=x_{0} \in \mathbb{R}$ then $S_{0}>0$.

We can rewrite equation (3.13) as

$$
\rho S u_{S S}=\frac{1}{4} \sqrt{\frac{S_{0}}{S}} e^{\frac{c t}{2}}+\frac{1}{\sigma^{2}}(c-r)-\frac{1}{4} .
$$

By localizing boundary conditions we get $\lim _{S \rightarrow \pm \infty} u_{S S}(S, t)=0$. Since

$$
\lim _{S \rightarrow \pm \infty} u_{S S}(S, t)=\lim _{S \rightarrow \pm \infty} \frac{1}{\sqrt{S}}=0
$$

equation (3.14) becomes $c=r+\frac{\sigma^{2}}{4}>0$ after simplifying.

Plugging this value of $c$ into (3.13) we obtain

$$
u_{S S}=\frac{\sqrt{S_{0}}}{4 \rho S^{3 / 2}} e^{\left(\frac{r+\sigma^{2} / 4}{2}\right) t} \quad \text { for } \quad \rho, S, S_{0}, \sigma>0, \quad \text { and } \quad r, t \geq 0 .
$$

Integrate (3.15) twice with respect to $S$. After a little algebra we get

$$
u(S, t)=a S-\frac{\sqrt{S_{0}}}{\rho}\left(\sqrt{S} e^{\left(\frac{r+\sigma^{2} / 4}{2}\right) t}+\frac{\sqrt{S_{0}}}{4} e^{\left(r+\sigma^{2} / 4\right) t}\right)
$$

for $S, S_{0}, \sigma, \rho>0, r, t \geq 0$ and $a \in \mathbb{R}$.

Substituting equation (3.16) into (2.5) and simplifying the results gives $a=1$. Since $a=1$ equation (3.16) reduces to (3.9). 
Remark 3.0.3. From equation (3.9), it is clear that $\frac{S u_{S}}{u}$ is always greater than one as in [2]. This shows that the option is always more volatile than the stock.

\section{The Greek Parameters}

We obtain the delta of the call option $u(S, t)$ by differentiating the solution (3.9) with respect to the spatial variable $S$. Hence,

$$
u_{S}=\frac{\partial u}{\partial S}=1-\frac{1}{2 \rho} \sqrt{\frac{S_{0}}{S}} e^{\left(\frac{r+\sigma^{2} / 4}{2}\right) t} \quad \text { for } \quad \rho, S, S_{0}, \sigma>0 \text { and } r, t \geq 0 .
$$

When $u_{S}$ is differentiated with respect to $S$ we obtain gamma as in equation (3.15).

The parameter theta is given by

$$
u_{t}=\frac{\partial u}{\partial t}=-\frac{\left(r+\sigma^{2} / 4\right) \sqrt{S_{0}}}{2 \rho}\left(\sqrt{S} e^{\left(\frac{r+\sigma^{2} / 4}{2}\right) t}+\frac{\sqrt{S_{0}}}{2} e^{\left(r+\sigma^{2} / 4\right) t}\right)
$$

for $\rho, S, S_{0}, \sigma, t>0$ and $r \geq 0$ when (3.9) is differentiated with respect to time $t$. If the price of the asset does not move, the option price will change by theta with time $t$.

Differentiating $u_{S S}$ with respect to $S$ gives option speed as

$$
u_{S S S}=\frac{\partial^{3} u}{\partial S^{3}}=-\frac{3 \sqrt{S_{0}}}{8 \rho S^{5 / 2}} e^{\left(\frac{r+\sigma^{2} / 4}{2}\right) t} \text { for } \quad \rho, S, S_{0}, \sigma>0 \text { and } r, t \geq 0 .
$$

Gamma is used by traders to estimate how much they will rehedge by if the stock price moves. An option delta may change by more or less the amount the traders have approximated the value of the stock price to change. If it is by a large amount that the stock price moves, or the option nears the strike and expiration, the delta becomes unreliable and hence the use of the speed.

When the solution (3.9) is differentiated with respect to $\sigma$ we get the vega of a call option $u(S, t)$ as

$$
\frac{\partial u}{\partial \sigma}=-\frac{\sigma t \sqrt{S_{0}}}{4 \rho}\left(\sqrt{S} e^{\left(\frac{r+\sigma^{2} / 4}{2}\right) t}+\frac{\sqrt{S_{0}}}{2} e^{\left(r+\sigma^{2} / 4\right) t}\right)
$$

for $\rho, S, S_{0}, \sigma>0$ and $r, t \geq 0$. 
The Greek parameter rho is obtained by differentiating (3.9) with respect to the interest rate $r$ to get

$$
\frac{\partial u}{\partial r}=-\frac{t \sqrt{S_{0}}}{2 \rho}\left(\sqrt{S} e^{\left(\frac{r+\sigma^{2} / 4}{2}\right) t}+\frac{\sqrt{S_{0}}}{2} e^{\left(r+\sigma^{2} / 4\right) t}\right)
$$

for $\rho, S, S_{0}, \sigma, r>0$ and $t \geq 0$.

\section{Conclusion}

We have studied the hedging of derivatives in illiquid markets. A model where the implementation of a hedging strategy affects the price of the underlying asset has been considered. Assuming the solution of a forward wave, a classical solution for the nonlinear Black-Scholes equation was found. The solution obtained can be used for pricing a European call option at time $t \geq 0$. This solution supports the comments in [2] that the option is always more volatile than the stock. All the risk parameters except Gamma are negative.

In conclusion, further research needs to be done to find solutions to arbitrary portfolio of options with non-single-signed gamma. Future work will also involve solving the nonlinear Black-Scholes equation using the hyperbolic tangent (tanh) method. We will also solve the Bakstein and Howison (2003) model in future.

\section{References}

[1] D. Bakstein, S. Howison, A non-arbitrage liquidity model with observable parameters for derivatives, Working paper (2003).

[2] F. Black, M. Scholes, The pricing of options and corporate liabilities, The Journal of Political Economy, 81, No. 3 (1973), 637-654, doi: $10.1086 / 260062$.

[3] U. Cetin, R. Jarrow, P. Protter, Liquidity risk and arbitrage pricing theory, Finance Stoch., 8 (2004), 311-341.

[4] J. Esekon, S. Onyango, N.O. Ongati, Analytic solution of a nonlinear Black-Scholes partial differential equation, International Journal of Pure and Applied Mathematics, 61, No. 2 (2010), 219-230. 
[5] J.E. Esekon, A particular solution of a nonlinear Black-Scholes partial differential equation, International Journal of Pure and Applied Mathematics, To Appear.

[6] J.D. Logan, An Introduction to Nonlinear Partial Differential Equations, Second Edition, John Wiley and Sons, Inc., Hoboken, New Jersey, USA (2008). 
